\title{
THE MEASUREMENT OF BLOOD PRESSURE
}

\author{
J. C. PATTERSON and J. M. FITZ-GERALD
}

(Received 10 January, 1975)

\begin{abstract}
In this paper, the procedure of the clinical measurement of blood pressure is modelled by the application of a uniform pressure band to a long, homogeneous, isotropic cylinder. The deformations are assumed to be infinitesimal, and transform methods are used to analyse the resulting equations. The inversion of the resulting transforms is carried out numerically. It is shown that, in spite of the fairly crude assumptions of the model, the actual load on the artery may be markedly different from that applied to the surface, leading to inaccuracies in the measured blood pressure. The parameter of importance is shown to be the ratio of pressure band width to arm diameter.
\end{abstract}

\section{Introduction}

On of the most common diagnostic procedures in clinical medicine is the measurement of blood pressure by direct, non-invasive means, of which by far the most common relies on the phenomenon of Korotkoff sounds, probably produced by a condition of dynamic instability of the brachial artery (Anliker and Raman [1]). The instability is the result of the application of a band of pressure to the outer surface of the upper arm by means of a pneumatic cuff, and the applied pressures at the onset and disappearance of the sounds are taken as estimates of the systolic and diastolic pressures respectively.

In the usual clinical situation, a standard width cuff is used for all patients, and the measured pressures compared with previous values. On the basis of this comparison, an estimate is made of the state (high or low) of the blood pressure. Except in a subjective sense, no account is taken of the difference between patients, particularly with respect to arm size; i.e. the effect of the tissue interposed between the point of application of the pressure and the artery itself is, in all cases, taken to be the same.

In this paper, a simple model of the situation is developed, and, in spite of the fairly crude assumptions involved, it is shown that the actual load at the 
artery position may depend quite significantly on the ratio of cuff length to arm diameter.

\section{The model and its analysis}

The upper arm is modelled by a long circular cylinder, the material of which is homogeneous and isotropic, no provision being made for the existence

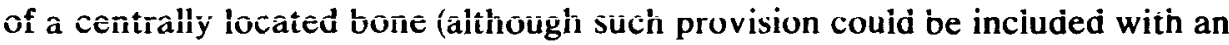
attendant increase in algebraic complexity). As well, the presence of the artery itself is ignored; a measure of the load on the artery is taken to be the radial stress at a point near the centre of the cylinder. The application of the pressurized cuff is assumed to be equivalent to the application of a band of normal stress over a section of the curved surface of the cylinder, the normal stress elsewhere on the surface being zero. The surface is assumed to be everywhere free of shear stress, and deformations are taken to be axisymmetric. Since flesh is almost incompressible, the Poisson's ratio $\nu$ of the material is given the value 0.45 .

In view of the assumptions made, the validity of applying any results to a real situation may be questionable. The assumptions of homogeneity and isotropy were, however, also used by Bennet [2] in a brief communication on the stress concentration effects of a prosthetic socket on limb stumps, the rationale being that a crude model may be sufficient to demostrate general behaviour in an initial study. The same is true here, and regardless of the inadequacies of the model, it is clearly demonstrated that a variation of stress does occur through the thickness of the arm, and that, for the same applied pressure, the radial stress near the centre depends markedly on the ratio of cuff length to arm diameter.

This particular problem was initially studied by Filon [3], who, by expanding the pressure band in terms of a Fourier series, was able to obtain approximate results for various problems involving finite length cylinders. A similar problem was analysed by Tranter and Craggs [5], using integral transform methods on an infinite cylinder loaded over half its length by a uniform pressure, later generalized by Sneddon [4] to include a non-uniform pressure. Similar problems, in which mixed conditions are prescribed on the surface, belong to the class of elastic contact problems, for which a vast literature exists. Here, consideration is limited to the solution of the model described, similar to the problem studied by Filon, by the transform methods of Sneddon.

The cylinder is taken to be of radius $a$, with Poisson's ratio $\nu$, and the normal pressure $p(z)$ (later taken to be uniform) is applied over the region $-b \leqq z \leqq b$, for $0 \leqq \theta \leqq 2 \pi$, in cylindrical polar coordinates $(r, \theta, z)$. The boundary conditions may then be written 


$$
\left.\begin{array}{rlrl}
T_{r z} & =0, & & \forall z \\
T_{r r} & =-p(z) & & |z| \leqq b \\
& =0 & & |z|>b
\end{array}\right\} \quad \text { on } r=a
$$

The stresses may be written in terms of a stress function, $\Phi$,

$$
\begin{aligned}
& T_{r z}=2 \mu \frac{\partial}{\partial r}\left((1-\nu) \nabla^{2} \Phi-\frac{\partial^{2} \Phi}{\partial z^{2}}\right) \\
& T_{r r}=2 \mu \frac{\partial}{\partial z}\left(\nu \nabla^{2} \Phi-\frac{\partial^{2} \Phi}{\partial r^{2}}\right)
\end{aligned}
$$

where $\mu$ is the shear modulus of the material, $\nabla^{2}$ the Laplacian operator in cylindrical polar coordinates, and

$$
\nabla^{2} \nabla^{2} \Phi=0
$$

If the Fourier transform $\bar{\Phi}$ of $\Phi$ is defined as

$$
\bar{\Phi}=\int_{-\infty}^{\infty} \Phi e^{i \xi z} d z
$$

with inverse

$$
\Phi=\frac{1}{2 \pi} \int_{-\infty}^{\infty} \bar{\Phi} e^{-i \xi z} d \xi,
$$

it is easy to show from the equations of equilibrium that

$$
\bar{\Phi}(\xi, r)=A_{1}(\xi) I_{0}(\xi r)+A_{2}(\xi) r I_{1}(\xi r),
$$

where, after use of the boundary conditions (1),

$$
A_{1}(\xi)=\frac{i \overline{\overline{p(\xi)}}\left[I_{0}(a \xi) a \xi+I_{1}(a \xi) 2(1-\nu)\right]}{\xi^{2}\left[\left(I_{0}(a \xi)\right)^{2} a \xi^{2}-\left(I_{1}(a \xi)\right)^{2}\left(\frac{2}{a}(1-\nu)+a \xi^{2}\right)\right]}
$$

and

$$
A_{2}(\xi)=\frac{-I_{1}(a \xi) A_{1}(\xi) \xi}{I_{0}(a \xi)+I_{1}(a \xi) 2(1-\nu)}
$$

with

$$
\bar{p}(\xi)=\int_{-b}^{b} \frac{p(z)}{2 \mu} e^{1 \xi z} d z
$$

The transform of the radial stress may then be written

$$
\bar{T}_{r r}(\xi, r)=2 \mu i \xi\left\{\begin{array}{c}
A_{1}(\xi)\left(\xi^{2} I_{0}(\xi r)-(\xi / r) I_{1}(\xi r)\right) \\
+A_{2}(\xi)\left(\xi(1-2 \nu) I_{0}(\xi r)+r \xi^{2} I_{1}(\xi r)\right)
\end{array}\right\} .
$$


For a uniform pressure, $p(z)=p,(9)$ gives

$$
\bar{p}(\xi)=\frac{1}{2 \mu} \frac{(2 p \sin (\xi b))}{\xi}
$$

and hence that

$$
T_{r r}=\frac{2}{\pi} \int_{0}^{\infty} F(r, \xi) \sin (L \xi) \cos (\xi z) d \xi
$$

with

$$
F(r, \xi)=\frac{\left[\begin{array}{c}
I_{1}(\xi)\left((1-2 \nu) I_{0}(r \xi)+r \xi I_{1}(r \xi)\right) \\
\left.-\left(I_{0}(\xi) \xi+I_{1}(\xi) 2(1-\nu)\right)\left(I_{0}(r \xi)-I_{1}(r \xi) / r \xi\right)\right)
\end{array}\right]}{\left.\left(I_{0}(\xi)\right)^{2} \xi^{2}-\left(I_{1}(\xi)\right)^{2}\left(2(1-\nu)+\xi^{2}\right)\right]} .
$$

where $\xi, r$, and $b$ have been non-dimensionalized with respect to $a$, and $T_{r r}$ with respect to $p$. Hence, the non-dimensional stress at may point $(r, z)$ may be calculated by evaluating the integral (12).

\section{Evaluation of the integral}

Although the integral (12) is merely a Fourier cosine transform, its inversion is not obvious, except in the case $r=1$, which yields the required boundary condition. For other values of $r$, the radial stress must be evaluated numerically.

The most direct procedure, since the integrand is regular on the real axis, appears to be numerical integration. Since the integrand oscillates with a frequency determined by the value of $z$, it was found that for large values of $z$ accuracy was somewhat limited. For small $z$, the oscillations are of period approximately $2 \pi / b$, and a ten point Gaussian integration routine over successive segments of length $2 \pi / b$ gave accurate results. As $z$ increased, the number of oscillations in each segment increased, and the accuracy suffered. It is, however, possible (in principle at least) to evaluate (12) for all values of $z$ by use of the calculus of residues.

It may be shown that, for realistic values of $\nu$, the poles of the integrand of (12) are all of single order, and that, in the region $\operatorname{Im} \xi>0$, they occur in pairs $\xi_{k}= \pm a_{k}+i d_{k}, k=1,2, \cdots$, with one pair of zeros corresponding to each pair of points $i j_{0 . k}, i j_{1 . k}$; in the usual notation $j_{\nu, k}$ is the $k$ th zero of $J_{\nu}(z)$, the Bessel function of order $\nu$. The conjugates are also poles. By a judicious choice of contour, (12) may be converted to a contour integral, and evaluated by the calculus of residues. For the case $z \geqq b$, the correct contour is the real axis and the lower semi-circle, and it is possible to show that the contribution from the pair of poles at $\xi= \pm a-i d$ is given by

$$
4 e^{-d z}\left[\begin{array}{c}
\sin b a \cosh b d(G \sin a z-H \cos a z) \\
+\cos b a \sinh b d(G \cos a z+H \sin a z)
\end{array}\right]
$$


where

$$
G+i H=\lim _{\xi \rightarrow a-i d}(\xi-(a-i d)) F(r, \xi) .
$$

The first four poles in the region $\operatorname{Re} \xi>0, \operatorname{Im} \xi<0$ were found numerically to be approximately

$$
\begin{aligned}
& \xi_{0}=1.3457-i \quad 2.7898 \\
& \xi_{1}=1.6353-i \quad 6.0862 \\
& \xi_{2}=1.8270-i \quad 9.2834 \\
& \xi_{3}=1.9665-i 12.4536
\end{aligned}
$$

and hence the contribution of further poles, with imaginary parts of greater magnitude, is insignificant, even for $z$ near $b$. In principle the integral may be evaluated exactly for $z \geqq b$ from (14) and (15); in practice, however, the evaluation of (15) is extremely sensitive to small errors in the determination of the poles. The behaviour for $z \geqq b$, is, nevertheless, demostrated by (14) to be an exponentially decaying oscillation, decreasing like $e^{\mathbf{- 2 . 8 z}}$ for sufficiently large $z$. With regard to the physiological problem, the region $|z|<b$ is of major interest; the behaviour in this region is calculated to sufficient accuracy by numerical integration.

\section{Results and discussion}

The integral (12) was evaluated for a range of values of $r, z$, and $b$. The value of $b$ is equivalent to the ratio of cuff length to arm diameter. Since $T_{r}$ is an even function of $z$, only positive values of $z$ are considered.

Figures 1-6 show the results of the numerical integration; Figures 1-3 showing the overall stress distribution with increasing $z$ for a number of radial positions and three different cuff lengths. Figures 4-6 demonstrate specific behaviour at the axial mid-point $z=0$. In the first group, the applied stress at $r=1$ is shown as a broken line.

Considering firstly Figure 1 , for a cuff length 0.5 , it is easily seen that near the centre $(r=0.1)$, the magnitude of the applied stress in the region $z<0.5$ is considerably less than unity, applied at $r=1$, whilst for $z>0.5$, the applied stress magnitude is greater than that applied at $r=1$ (zero). Hence, for a relatively narrow cuff, some "leakage" of stress from beneath the cuff has occurred. The same is also true to a lesser degree at $r=0.5$ and $r=0.9$, the effect diminishing as the distribution curves tend to the step function to meet the boundary condition at $r=1$. A slight expansionary stress $\left(T_{r}>0\right)$ is present for each radius for a range of $z$ above $z=0.5$; again this effect is most 


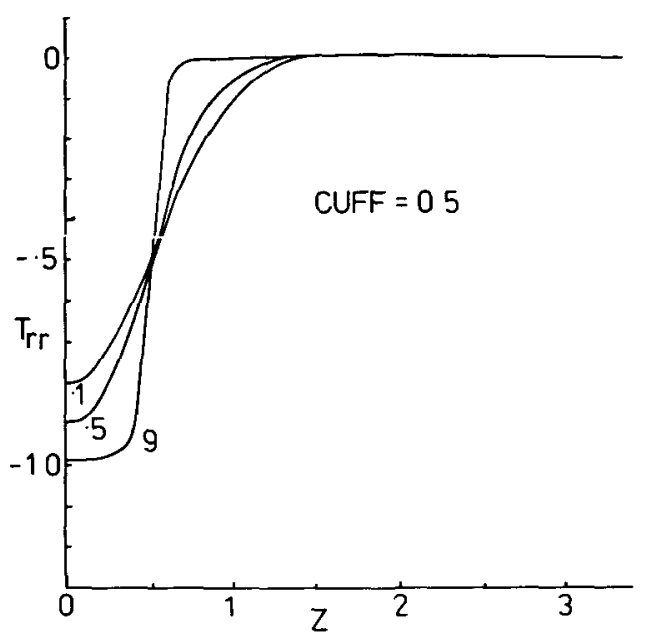

Figure 1. The variation of the radial stress $T_{r r}$ with axial distance $z$ for radii $0.1,0.5$, and 0.9 with a cuff length of 0.5 .

obvious at $r=0.1$, being barely present at $r=0.9$. This expansion stress will cause the expected bulging of the cylinder past the ends of the cuff.

For a cuff length of 1.0 (Figure 2 and 2(a)), the behaviour is generally similar, with the exception that the magnitude of the stress at $z=0$ is now greater than unity for $r=0.1$ and $r=0.5$ by about $1 \%$, whilst at $r=0.9$, it

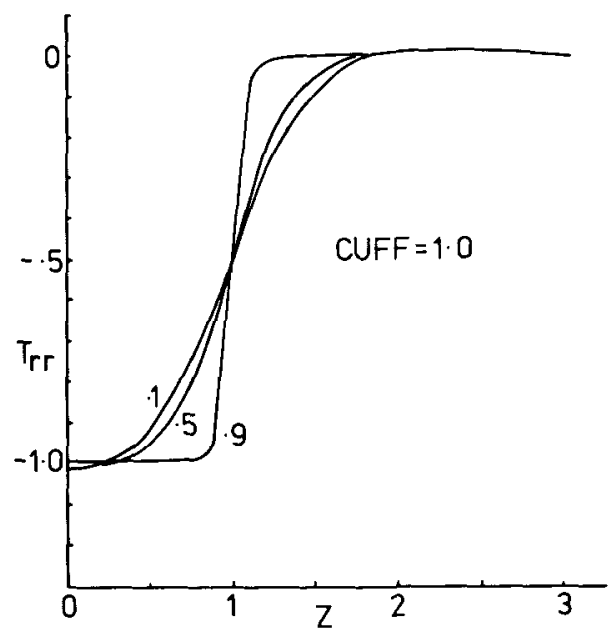

Figure 2. The variation of the radial stress $T_{r r}$ with axial distance $z$ for radii $0.1,0.5$, and 0.9 with a cuff length of 1.0 . 


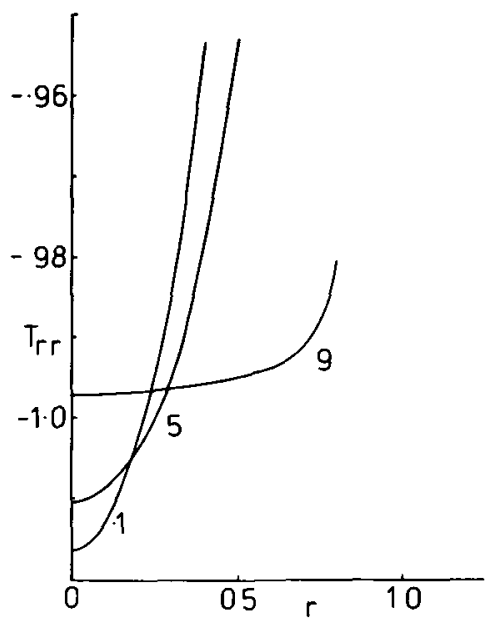

Figure 2(a). The radial stress $T_{r r}$ for a cuff length of 1.0 on an expanded scale for $0 \leqq z \leqq 1$.

is marginally less than unity. Hence, a leakage does not appear to have occurred; rather a stress concentration at the centre seems apparent. This effect is discussed further in the light of Figures 3 and 3(a) for a cuff length of 2.0. Here, the stresses at $z=0$ do not have the maximum magnitude, the maximum being reached at a point slightly less than $z=0.8$, for the lower values of $r$ at least. At $r=0.9$, the magnitude falls smoothly off to zero with

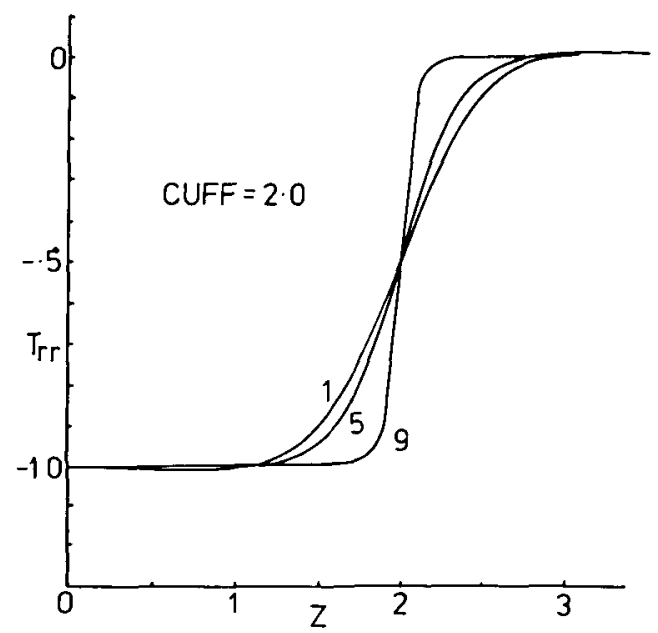

Figure 3. The variation of the radial stress $T_{r}$ with axial distance $z$ for radii $0.1,0.5$, and 0.9 with a cuff length 2.0. 


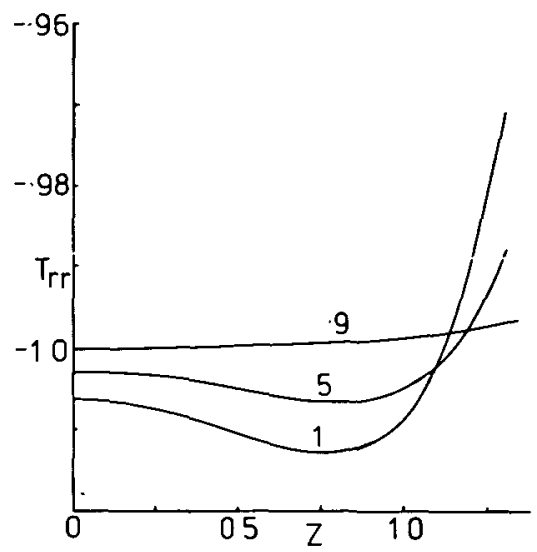

Figure 3(a). The radial stress $T_{r}$ for a cuff length of 2.0 on an expanded scale for $0 \leqq z \leqq 1.25$.

increasing $z$. This maximum is greater in magnitude than unity, and is presumably in some way related to the expansionary stress outside the cuff. The concentration effect for a cuff length of unity is the result of the combining of the maxima from each end of the cuff. This maximum magnitude was present for all cuff lengths greater than 2.0 used in the calculations, although the result for 2.0 only is shown. In all cases, however, the maximum occurred at a distance slightly greater than 1.2 inside the cuff. Hence it would be expected that the absolute maximum of the stress magnitude at $z=0$ would occur at a cuff length near 1.2, where the maxima from each end would reinforce to the greatest extent.

It is not difficlt to suggest a physical explanation of these results. The bulging of the material just past the end of the cuff is the result of a stress concentration just inside the cuff, as mentioned in connection with Figure 3. For long cuffs, this gives rise to a boundary layer effect, extending over a region of about one diameter in length. For decreasing cuff size, these boundary layers eventually take over the entire region, with a reinforcement effect as mentioned. For smaller cuffs, "leakage" begins to predominate, and the stress concentration effect occurs closer to the surfaces. In fact, for cuff lengths between 0.75 and 0.9 , the maximum stress occurs (at $z=0$, as expected) for increasing $r$ as cuff length decreases, until at 0.75 , the stress magnitude simply decreases in all directions away from the cuff.

This reinforcement effect is borne out by Figure 4, which shows the normal stress as a function of cuff length for a typically small radius $(r=0.1)$. For a cuff length near zero, the applied stress near the centre is naturally very small, as the actual load is also near zero. With increasing cuff length, the stress 


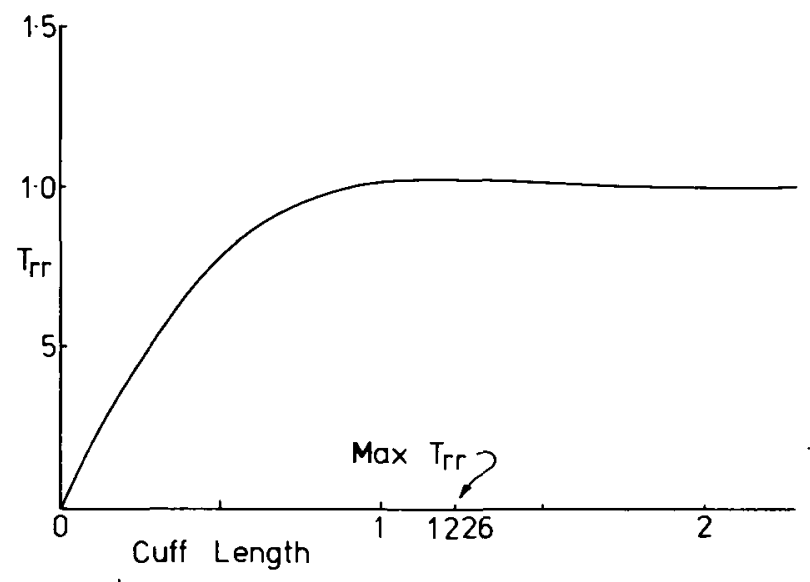

Figure 4. The variation of radial stress $T_{r}$ at $z=0, r=0.1$, with cuff length.

magnitude rises fairly steeply, before flattening out and reaching a maximum at a cuff length of 1.226 , close to the value predicted above. With increasing cuff length, the stress asymptotically approaches unity, as expected.

Figure 5 and 6 show the variation of stress with radius at $z=0$ for various cuff lengths; Figure 5 detailing the behaviour for cuff lengths $0.25,0.5$, and 1.0, and Figure 6 for cuff lengths 1.0 and 2.0, on an expanded scale. For small cuff lengths the stress magnitude near the centre is small, as noted earlier, increasing with radius to unity at $r=1$, as required. As the cuff length

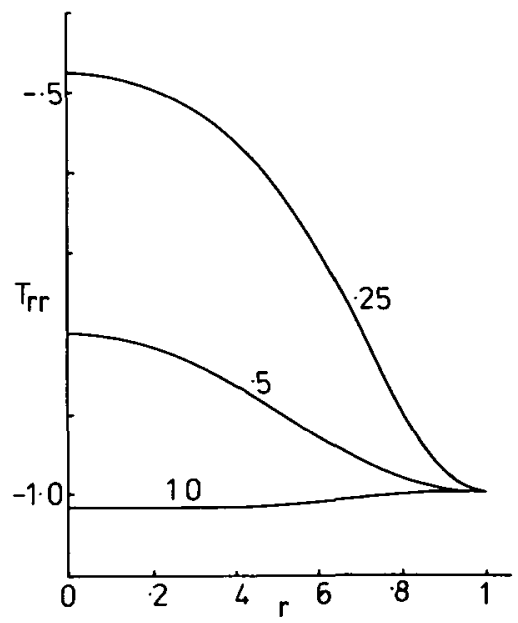

Figure 5. The variation of radial stress $T_{m}$ at $z=0$ with radius for cuff lengths of $0.25,0.5$, and 1.0. 


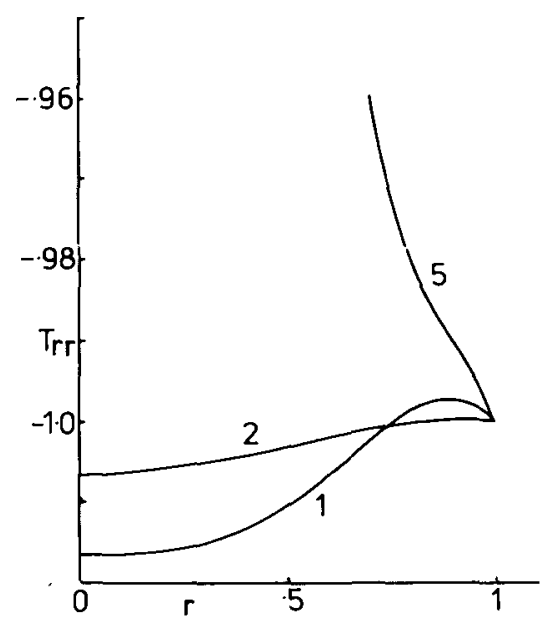

Figure 6. The variation of radial stress $T_{r}$ at $z=0$ with radius for cuff lengths of $0.5,1.0$, and 2.0, on an expanded scale.

increases, so does the stress magnitude at $r=0$, reaching a maximum for a cuff of length 1.226, the curve for this value not being shown in the interests of clarity. For a cuff length of 1.0 , the stress magnitude is greater than unity for $r<0.76$, but less than unity for $0.76<r<1.0$, a minimum being reached at $r=0.88$. Hence, in this region, the material is less "compresed" than at the centre. The effect is not present for cuff lengths of $\mathbf{2 . 0}$ or greater. For these larger values, the curves flatten out and approach unity everywhere with increasing cuff length. For the longest cuff considered, with a length of 5.0, the stress was in fact indistinguishable from unity at all values of $r$.

The implications with regard to the measurement of blood pressure are obvious; as a standard width cuff is used, the measure of pressure depends on the diameter of the subject's arm. If the magnitude of the normal stress at $r=0.1$ is taken as a measure of the actual pressure applied to the artery, and the cuff length is equal to the diameter of the arm, the closing pressure will be understimated by a factor of $1.6 \%$ approximately. The effect decreases for increasing cuff length; however, it increases sharply for decreasing cuff length. For example, an arm such that the cuff length is 0.75 of the diameter will cause an overestimation of the order of $5 \%$, whilst if the ratio is 0.5 , the overestimation will be of the order of $20 \%$. As the measurement of blood pressure is indirect, and its results compared with previous measurements on different subjects, it is plausible to suggest that extremely fleshy patients may well be suspected of slight hypertension which does not in fact exist.

Before any real quantitative predictions are made, several improvements 
to the model should be incorporated, the most obvious of which are the inclusion of a centrally located bone and more realistic boundary conditions at the surface. These improvements, however, are unlikely to significantly alter the main points of the simple model; that the radial stress does vary through the arm, and that the variation depends markedly on the ratio of cuff length to arm diameter.

\section{References}

[1] M. Anliker and K. R. Raman, "Korotkoff sounds at diastole - a phenomenon of dynamic instability of fluid-filled shells", Int. J. Solids Structures, 2 (1966), 467-491.

[2] L. Bennet, Discussion in Perspectives in Biomedical Engineering, Proceedings of a Symposium at the University of Strathclyde (Ed. by R. M. Kenedi), MacMillan Press Ltd., London (1973), 224-227.

[3] L. N. G. Filon, "On the elastic equilibrium of circular cylinders under certain practical system of load", Philos. Trans. Roy. Soc. Lond. Sec. A 198 (1902), 147-233.

[4] I. N. Sneddon, Fourier Transforms, McGraw-Hill, New York (1951).

[5] C. J. Tranter and J. W. Craggs, "The stress distribution in a long circular cylinder when a discontinuous pressure is applied to the curved surface", Philos. Mag., 36 (1945), 241-250.

Department of Mathematics

University of Queensland

St. Lucia, QLD.

Australia. 